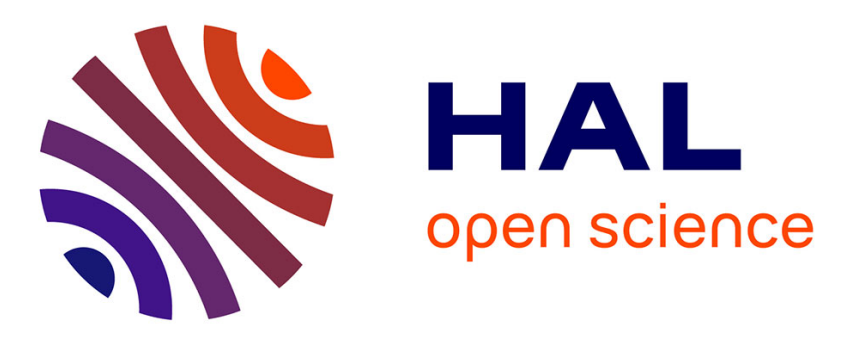

\title{
Hyperintensity of functional networks involving voice-selective cortical regions during silent thought in schizophrenia
}

Lucile Rapin, Marion Dohen, Hélène Loevenbruck, Jen Whitman, Paul

Metzak, Todd S. Woodward

\section{To cite this version:}

Lucile Rapin, Marion Dohen, Hélène Loevenbruck, Jen Whitman, Paul Metzak, et al.. Hyperintensity of functional networks involving voice-selective cortical regions during silent thought in schizophrenia. Psychiatry Research: Neuroimaging, 2012, 202 (2), pp.110-117. 10.1016/j.pscychresns.2011.12.014 . hal-00734043

\section{HAL Id: hal-00734043 \\ https://hal.science/hal-00734043}

Submitted on 29 Jan 2013

HAL is a multi-disciplinary open access archive for the deposit and dissemination of scientific research documents, whether they are published or not. The documents may come from teaching and research institutions in France or abroad, or from public or private research centers.
L'archive ouverte pluridisciplinaire HAL, est destinée au dépôt et à la diffusion de documents scientifiques de niveau recherche, publiés ou non, émanant des établissements d'enseignement et de recherche français ou étrangers, des laboratoires publics ou privés. 
Running head: Voice-Selective Cortical Regions

Hyperintensity of functional networks involving voice-selective cortical regions during silent thought in schizophrenia

Lucile A. Rapin ${ }^{\mathrm{a}, \mathrm{b}, \mathrm{c}}$, Marion Dohen ${ }^{\mathrm{a}}$, Hélène Lœevenbruck ${ }^{\mathrm{a}}$, Jennifer C. Whitman ${ }^{\text {b,c }}$, Paul D. Metzak ${ }^{\text {b,c }} \&$ Todd S. Woodward ${ }^{\text {b,c,* }}$

${ }^{\mathrm{a}}$ Speech and Cognition Department, GIPSA-lab, UMR5216, CNRS, Grenoble University, Grenoble, France

${ }^{\mathrm{b}}$ Department of Psychiatry, University of British Columbia, Vancouver, Canada

${ }^{c}$ Department of Research, BC Mental Health \& Addictions Research Institute, Vancouver Canada

${ }^{*}$ Corresponding author. Please address correspondence to Todd S. Woodward, Ph.D., BC Mental Health \& Addictions Research Institute, Room 116, 3rd Floor, 938 W. 28th Avenue, Vancouver, B.C., Canada. V5Z 4H4. e-mail: todd.woodward@ubc.ca. Phone: 604-875-2000 x 4724. Fax: 604-875-3871.

Abstract: 166 words $\quad$ Text Body: 5170 words 
Abstract

An important aspect of schizophrenia symptomatology is inner-outer confusion, or blurring of ego boundaries, which is linked to symptoms such as hallucinations and Schneiderian delusions. Dysfunction in the cognitive processes involved in the generation of private thoughts may contribute to blurring of the ego boundaries through increased activation in functional networks including speech- and voice-selective cortical regions. In the present study, the neural underpinnings of silent verbal thought generation and speech perception were investigated using functional magnetic resonance imaging (fMRI). Functional connectivity analysis was performed using constrained principal component analysis for fMRI (fMRI-CPCA). Group differences were observable on two functional networks: one reflecting hyperactivity in speech- and voice-selective cortical regions (e.g., bilateral superior temporal gyri; STG) during both speech perception and silent verbal thought generation, and another involving hyperactivity in a multiple demands (i.e., task-positive) network that included Wernicke's area, during silent verbal thought generation. This set of preliminary results suggests that hyperintensity of functional networks involving voice-selective cortical regions may contribute to the blurring of ego boundaries characteristic of schizophrenia.

KEYWORDS: SPEECH PERCEPTION; INNER THOUGHTS; COVERT SPEECH; FUNCTIONAL MAGNETIC RESONANCE IMAGING; FUNCTIONAL CONNECTIVITY 


\section{Introduction}

An important aspect of schizophrenia symptomatology is alienation, which can lead to self-other and/or inner-outer confusion, or blurring of ego boundaries, and is linked to symptoms such as hallucinations and Schneiderian delusions (Larøi and Woodward, 2007; Waters et al., 2010). In the source monitoring and schizophrenia literature (Seal et al., 2004; Woodward et al., 2007; Allen et al., 2008), it has been suggested that alienation and misattribution of thoughts could be explained by either a degraded top-down "generatingthoughts" signal, or the bottom-up addition of perceptual attributes to internal events. We investigated this issue using functional magnetic resonance imaging (fMRI) to study the cognitive operations accompanying the formation of private, self-generated thoughts as well as speech perception.

Examination of the cognitive operations contributing to thought generation and speech perception can be enhanced using fMRI because the neural regions involved in the cognitive operations of theoretical interest are relatively well established. Specifically, the generation of thoughts would be expected to activate what is referred to as the task-positive (Fox et al., 2005) or multiple demands network (Duncan and Owen, 2000), involving the dorsal frontal, parietal, and anterior cingulate cortices, as these activate when attentionally demanding tasks are carried out. In comparison, speech perception should activate primarily speech- and voiceselective cortical regions (Belin et al., 2000), and more specifically vocal speech regions, which include the primary auditory cortex, superior temporal gyrus (STG) and adjacent Sylvian fissure regions (Zatorre et al., 1992; Belin et al., 2000; Binder et al., 2000; Buchsbaum et al., 2005; Hickok and Poeppel, 2007). Thus, measurement of the functional networks involved in thought generation and speech perception in schizophrenia patients could provide evidence that relates to alienation theories. 
Early attempts at localizing the neural underpinnings of silent thought employed overt and/or covert word generation. However, overt generation of a word (Frith et al., 1991; Frith et al., 1995; Friedman et al., 1998) does not promote the separation of perceptual and generational cognitive processes. Studies using covert generation of a word (McGuire et al., 1996a; Curtis et al., 1998) allowed generation to be studied separately from perception, but perception of another's voice for comparison was omitted from these studies. One positron emission tomography (PET) study on healthy subjects employed sentence generation and a separate speech perception condition (Müller et al., 1997). The results indicated that sentence generation activated left dorsolateral prefrontal cortex (DLPFC; BA 46), whereas sentence perception activated bilateral superior temporal regions (BA 22), suggesting a separation of top-down effortful processing and bottom-up speech perception. However, only overt speech was used in the sentence generation condition in this study as well, thereby introducing a perceptual component to the generation condition.

In the current study, a new fMRI paradigm with two conditions was developed in order to separate silent verbal thought generation from speech perception. In the silent verbal thought generation condition, participants were required to generate a definition of a common word when presented with an illustration of the object that the word represents. In the speech perception condition, participants were required to listen to a definition of a common word when presented with an illustration of the object that the word represents. We used an analysis method that allowed us to study networks of coordinated brain activity as opposed to activation in individual regions: constrained principal component analysis (CPCA) for fMRI (fMRI-CPCA; www.nitrc.org/projects/fmricpca) with a finite impulse response (FIR) model (Metzak et al., 2011a; Metzak et al., 2011b). This method of analysis allows not only the visualization of how functional networks respond to verbal thought generation and speech perception, but also visualization of how activation and deactivation in these connected 
networks differ between healthy controls and schizophrenia patients for these specific tasks. As with univariate methods, deactivation refers to network regions that decrease activity during task-on periods relative to task-off periods, and activation refers to regions that increase activity during task-on periods relative to task-off periods.

According to top-down and bottom-up accounts of alienation in schizophrenia, if alienation were due to increased bottom-up perceptual qualities associated with silently generated thoughts, an increased intensity in functional networks involving voice-selective cortical regions would be expected during silent generation of thoughts in the schizophrenia patients relative to healthy controls. In contrast, if alienation were due to decreased intensity of the top-down "generating thoughts" signal, decreased activity in the cognitive control (multiple demands) functional network may be present in the schizophrenia patients relative to the healthy controls during silent generation of thought. Either result would contribute to theoretical accounts of how inner-outer confusion and alienation arise in schizophrenia. The speech perception condition was included as a control/comparison condition, and no specific hypotheses were put forward for this condition.

\section{Methods}

\subsection{Participants}

Five schizophrenia patients and ten healthy controls were examined. All the participants were right-handed (Annett, 1970) and all had been using English daily for at least the past 5 years. The patient group was composed of 3 males and 2 females who were recruited from the Vancouver General Hospital wards located at the University of British Columbia. All diagnoses were based on DSM-IV-R criteria (Association, 2000), and were made by a multidisciplinary team conference during the first month of admission when diagnosis is reviewed using all sources of information. Four patients were diagnosed with schizophrenia and one with schizoaffective disorder. Diagnoses were confirmed at the time of 
testing using the Mini-International Neuropsychiatric Interview (MINI; Sheehan et al., 1998). All patients were taking atypical neuroleptics at the time of study. Two patients were taking Risperidone (mean dosage $=3 \mathrm{mg} /$ day, range $=2-4 \mathrm{mg}$ ), one was taking Clozapine (600mg/day), one Quetiapine (600mg/day) and one Fluephenazine (40mg/day). The mean duration of illness was 8.2 years $(\mathrm{SD}=4.38)$. The control group was composed of 5 male and 5 female healthy volunteers who were recruited from advertisement posts. The exclusion criteria for both groups included (1) history of neurological disorder, traumatic brain injury with loss of consciousness for more than 5 minutes or any cognitive sequalae resulting from loss of consciousness, and (2) drug/alcohol abuse or addiction. History of psychiatric disorder (self or close family) warranted exclusion for the control group, and patients with strong negative symptoms or severe thought disorganisation symptoms were excluded. IQ was evaluated using the Kaufmann Brief Intelligence Test version 2 (KBIT-2; Kaufman and Kaufman, 1997) and the Wechsler Test of Adult Reading (WTAR; Corporation, 2001). With respect to the KBIT-2, the mean score for the control group was $110.50(\mathrm{SD}=9.58)$ and the mean score for the patient group was $98.6(\mathrm{SD}=16.42)$. With respect to the WTAR test, the mean score for the control group was $111.5(\mathrm{SD}=6.50)$ and the mean score for the patient group was 104.6 $(\mathrm{SD}=6.78)$. T-tests performed on the mean scores suggested no significant difference between the two groups on the KBIT-2, $t(13)=1.79, P=0.1$, or the WTAR, $t(13)$ $=1.56, P=0.14$. The mean age was 33.6 years $(\mathrm{SD}=7.76)$ for the patients and 26.7 years $(\mathrm{SD}=7.54)$ for the controls, and there was no significant age difference between the two groups, $t(13)=1.65, P=0.12$.

The Signs and Symptoms of Psychotic Illness (SSPI; Liddle et al., 2002) scale was used to measure the symptom history as well as the severity of the illness over the past week. SSPI is a 20 item scale with a 5 point range $(0=$ no symptoms; $4=$ severe symptoms $)$. The mean SSPI score for the schizophrenia patients was $11(\mathrm{SD}=6.04)$ consistent with moderate 
symptomatology (Liddle et al., 2002). One patient had hallucinated in the past week, but no others reported hallucinations or Schneiderian delusions. A hearing test was performed on all the participants using an audiometer (AMBCO 650AB, www.ambco.com) to ensure absence of hearing impairment. All subjects gave written informed consent and met fMRI compatibility criteria. The study was approved by Clinical Research Ethics Committees of the University and Hospital. Participants received a financial reward of \$10 CAD per hour for participation in the study.

\subsection{Conditions}

Two experimental conditions were examined: silent verbal thought generation and speech perception. In the silent verbal thought generation condition, participants were required to mentally generate a simple definition of a word, with the word and corresponding object visually presented on a screen. For example, the word "Table" would appear along with a picture of a table, and participants may generate the definition "Something you eat meals on." In the speech perception condition, participants listened to a simple definition of a word starting with "Something you...", with the word and corresponding object visually presented on the screen. An example of a typical stimulus is shown in Figure 1. Participants were instructed that each definition, whether heard or generated, should start with "Something you" in order to ensure that participants would generate at least some words on every trial, and to minimize any interpretational confounds between conditions.

\subsection{Stimuli}

Three 30-word lists of nouns were created using the MRC database (Coltheart, 1981). In order to facilitate the generation of definitions, only nouns with values greater than 500 on familiarity, concreteness and imageability criteria ratings were chosen (700 being the

maximum value), and the three lists were matched by mean values on these parameters. All nouns were of neutral affective content and included the categories of food, houseware, 
furniture, clothing and transportation devices. Extensive pilot testing was carried out to ensure that the nouns included in the experiment evoked consistent definitions, and that when presented with definitions only, the intended target word was produced. The audio stimuli were recorded by a female native speaker of English in a quiet room. The recorded definitions lasted on average 2.22 seconds $(S D=0.62)$. Two out of the three word lists were randomly assigned to the two conditions for each participant separately.

\subsection{Design and Procedure}

Prior to the fMRI scans, the subjects were familiarized with the experimental procedure (both conditions) in a computerized practice run, using 10 words different from those presented in the scanner. Following this, in order to facilitate generation of definitions while in the scanner, participants practiced audibly generating definitions for the 30 -word list in the generation condition, with no time limit imposed. For each word presented on the screen they were asked to overtly generate a simple definition of the word starting with "Something you ...". Once in the scanner, the experimenter reminded participants about the silent generation condition, and instructions were presented on the screen to ensure the task was performed covertly, and within the time allotted for each response. It was emphasized that participants should avoid generating their own definitions when one was provided (i.e., during the speech perception condition). No specific training was carried out for the speech perception material, to ensure that familiarity with the material would not increase the likelihood of self-generation of definitions during the speech perception condition.

During fMRI scanning, the two experimental conditions were presented using Presentation Software 12.1 (http://www.neurobs.com). For each condition, each word and its associated illustration were presented (in a different randomized order for each subject) for two seconds. A blank screen was then shown for three seconds. Total trial duration was five seconds. Words were written in white in a 48 point Times New Roman font and presented on 
a black background. The illustration was placed under the word and the display was centred on the screen (see Figure 1). At the end of each condition block, there was a rest break during which the participants were required to do nothing other than remain still for 60 seconds. In the speech perception condition, the onset of each audio file was synchronized to visual presentation of the corresponding word.

In order to prevent subjects from internally reviewing the most recently generated or heard definition (which would act to reduce the power of the experimental manipulation), during the interstimulus interval (ISI) a display of generic circles moving in an "orbiting" motion was presented. The distribution of the duration of the ISI was exponential, optimizing the deconvolution of the BOLD signal (Serences, 2004, mean $=4.46 \mathrm{~s}$, range $=2 \mathrm{~s}, 4,6,8,16$, 20s). Two functional scan runs of 9 minutes and 25 seconds each were completed, and stimuli in each condition were grouped in blocks consisting of fifteen trials in each run. The order of the conditions (as well as the words within the conditions) was randomly assigned.

\subsection{Image Acquisition}

Echo-planar functional MR images were collected with a Philips Achieva 3.0 Tesla MRI scanner Parallel imaging was used with a SENSE method ( $\mathrm{AP}=2$; os $=1)$. The main acquisition parameters were: TR of $2.5 \mathrm{~s}$, TE of $30 \mathrm{~ms}$, flip angle $90^{\circ}, 80^{*} 80$ voxels, field of view $240 \mathrm{~mm}$ (voxel size $3 * 3 * 3 \mathrm{~mm}$ ). 440 images were acquired in two runs of $9.25 \mathrm{~min}$ each. Each whole brain volume consisted of 36 transverse interleaved slices parallel to the bicommissural plane (slice thickness: $3 \mathrm{~mm}$, interslice gap: $1 \mathrm{~mm}$ ). Prior to the functional scans, an anatomical whole brain MR scan was obtained.

\subsection{Image Analysis}

Functional images were first pre-processed using SPM5 (Wellcome Institute of London, UK). Images were realigned, normalized to the Montreal Neurological Institute 
(MNI) brain template and were spatially smoothed with an $8 * 8 * 8 \mathrm{~mm}$ full width at half maximum Gaussian filter.

Data analysis was carried out using Constrained Principal Component Analysis for fMRI (fMRI-CPCA; http://www.nitrc.org/projects/fmricpca) with an orthogonal rotation (Metzak et al., 2011a; Metzak et al., 2011b). The theory and proofs of CPCA are detailed in previously published work (Takane and Shibayama, 1991; Takane and Hunter, 2001). Briefly, CPCA combines principal component analysis and regression analysis. When applied to fMRI data, three main steps are carried out. In the first step, referred to as the external analysis, a multivariate least-square multiple regression is carried out to separate the BOLD signal into variance that is and is not predictable from stimulus timing model (the FIR model - FIR response models do not assume a BOLD signal shape, but estimate BOLD changes in peristimulus time). In the second step, the predicted scores from the multivariate multiple regression are submitted to principal component analysis (PCA with rotation). In a third step, weights are computed that, when applied to the stimulus timing model, would produce the component scores (the latter run over scans and subjects, as does the FIR model). This produces subject-, condition-, and perstimulus-time-point-specific predictor weights for each functional network, which reflect the intensity of each functional nework over peristimulus time, and as such, estimate the hemodynamic response (HDR) associated with each functional network. Predictor weights can be interpreted as the importance of each extracted component to each combination of peristimulus time point, subject and scan.

\subsection{Statistical Inference Procedure}

Predictor weights can be used to test the effects of the experimental condition on estimated BOLD response and to compare these responses between groups (Metzak et al., 2011a; Metzak et al., 2011b). These analyses were carried out as $7 \times 2 \times 2$ mixed-model ANOVAs, with the within-subjects factors of Peristimulus Time ( 8 whole-brain scans after 
the onset of each stimulus were estimated in the FIR model, but the first value is set to zero for all subjects and conditions so is not included in the ANOVA) and Condition (speech perception vs. thought generation), and the between-subjects factor of Group (controls vs. patients). Greenhouse-Geisser adjusted degrees of freedom were checked, but any violations of sphericity did not affected interpretation of results; therefore, the original degrees of freedom are reported. Predictor weights provide estimates of the intensity of functional networks over perstimulus time, and as such, estimate the HDR associated with each functional network; therefore, a significant effect of peristimulus time combined with a biologically plausible HDR shape provides evidence that the component is reflecting a reliable BOLD response to the stimuli.

\section{Results}

Inspection of the scree plot (Cattell, 1966, 1977) of singular values obtained from the CPCA analysis suggested that six components should be extracted. All six components were tested to ensure that (1) they took the form of a HDR shape, and (2) showed a statistically significant effect of peristimulus time (see Metzak et al., 2011a; Metzak et al., 2011b). The first component, reported in Supplementary Material - Table 1 and displayed in Figure 2A, accounted for $12.79 \%$ of the rotated variance predictable from stimulus presentation timing. It included a network of activations in bilateral visual regions and fusiform gyri (BAs 18, 19), bilateral dorsal anterior cingulate/supplementary motor area (BA 6), bilateral prefrontal regions (BAs 45, 46, 47), bilateral insular cortices, and Wernicke's area (BA 22; posterior STG). It showed significant main effects of Peristimulus Time, $F(6,78)=4.14, P<0.01$, Condition, $F(1,13)=15.31, P<0.01$, and Group, $F(1,13)=4.57, P=0.05$. However, a significant interaction between Peristimulus Time and Condition, $F(6,78)=5.20, P<0.001$, was also observed. The source of this interaction was that the estimated BOLD response was stronger in the thought generation condition, $F(6,78)=9.03, P<0.001$, than in the speech 
perception condition, $F(6,78)=0.46, P=0.83$, leading to a higher peak, and a HDR shape that differs between conditions. The significant group effect in the absence of interactions suggests that, averaged over conditions and time-points, the activity in this network was stronger in patients $($ mean $=0.15)$ than in controls $($ mean $=-0.03)$, but to interpret this it must be kept in mind that the component is mostly influenced by the thought generation condition.

The second component, reported in Supplementary Material - Table 2 and displayed in Figure $2 \mathrm{~B}$, accounted for $9.86 \%$ of the variance predictable from stimulus presentation timing. It comprised a functional network with activations in bilateral STG (BAs 21, 22, 41) and fusiform gyrus/occipital/cerebellar regions (BAs 17, 18, 19). This component was characterized by a significant main effect of Peristimulus Time, $F(6,78)=4.48, P<0.001$, but also a significant interaction between Condition and Peristimulus Time, $F(6,78)=4.96, P$ $<0.001$. This interaction indicates that the shape of the estimated HDR depended on the condition. Repeated contrasts show that this interaction was strongest for changes over the 9 second, $F(1,13)=9.59, P<0.01$, and 15 second, $F(1,13)=10.99, P<0.01$, time-points, reflective of an increase and decrease in estimated HDR (i.e., higher peak) in the speech perception condition that is absent in the thought generation condition, which is congruent with involvement of the STG.

The third component, reported in Supplementary Material - Table 3 and displayed in Figure 2C, accounted for $7.29 \%$ of the variance predictable from stimulus presentation timing. It was defined by a functional network of activations in the lingual gyrus/occipital/cerebellar regions (BAs 17, 19), bilateral STG (BAs 21, 22), dorsal anterior cingulate/supplementary motor area cortex (BA 8) and the left inferior frontal and dorsolateral prefrontal cortices (BAs $45,46,47)$. This component was characterized by a main effect for Peristimulus Time, $F(6$, $78)=27.81, P<0.001$, and an interaction between Peristimulus Time and Condition, $F(6,78)$ $=7.18, P<0.001$. The interaction was significant only for changes over the 15 second time- 
point, $F(1,13)=24.49, P<0.001$, which was characterized by a decline in activation for speech perception and an increase for generation, caused by a slightly later peak for thought generation.

The fourth component, reported in Supplementary Material - Table 4 and displayed in Figure 2D, accounted for $6.50 \%$ of the variance predictable from stimulus presentation timing. It was characterized by a functional network with activations in the lingual gyrus/occipital/cerebellar regions (BA 17, 18, 19), and the left superior frontal and precentral (BA 6) regions. It also included deactivations in the bilateral middle (BA 8) and right inferior (BAs 10, 47) frontal regions as well as in the angular and supramarginal gyri (BAs 39, 40). This component showed significant main effects of Peristimulus Time $F(6,78)=23.14, P<$ 0.001, and of Condition, $F(1,13)=9.54, P<0.01$. The Peristimulus Time $\times$ Condition interaction was also significant, $F(6,78)=14.00, P<0.001$, indicating that the shape of the HDR is condition dependent. The interaction was especially strong for changes over the 9 second and 18 second time windows, $F(1,13)=26.52, P<0.001 ; F(1,13)=71.09, P<0.001$, due to the steeper incline and decline for the thought generation condition relative to the speech perception condition.

The fifth component, reported in Supplementary Material - Table 5 and displayed in Figure 2E, accounted for $4.17 \%$ of the variance predictable from stimulus presentation timing. It involved a functional network with activations in the bilateral STG (BAs 21, 22) and deactivations in the bilateral medial and anterior frontal regions (BAs 10, 32), and posterior cingulate gyrus (BAs 7, 31). This component showed significant main effects of Peristimulus Time $F(6,78)=13.43, P<0.001$, and of Condition, $F(1,13)=14.80, P<0.005$. The interaction between these factors was also significant, $F(6,78)=6.51, P<0.001$. The interaction was especially strong for changes over the 9 second and 18 second time windows, $F(1,13)=27.31, P<0.001 ; F(1,13)=11.91, P<0.005$, due to the steeper incline and decline 
for the speech perception condition relative to the thought generation condition, and possibly a later peak for the thought generation condition.

The sixth component, reported in Supplementary Material - Table 6 and displayed in Figure $2 \mathrm{~F}$, accounted for $3.63 \%$ of the variance predictable from stimulus presentation timing. It was characterized by a functional network with activations in the bilateral STG (BAs 21, 22) as well as in the lingual gyrus/fusiform gyrus/occipital/cerebellar regions (BAs 18, 19). It was also comprised of deactivations mainly in the bilateral superior frontal regions (BAs 9, $45,46,47)$ and the angular and supramarginal gyri (BAs 39, 40). This component showed significant main effects of Peristimulus Time $F(6,78)=37.55, P<0.001$, and of Condition, $F(1,13)=20.06, P<0.001$, as well as a significant Condition $\times$ Peristimulus Time interaction, $F(6,78)=10.18, P<0.001$. The interaction was especially strong for changes at four time points from 9 to 18 seconds, $F(1,13)=52.05, P<0.001, F(1,13)=28.07, P<$ $0.001 ; F(1,13)=63.18, P<0.001 ; F(1,13)=24.38, P<0.001$. This is due to the sharp peak for the speech perception condition relative to thought generation, which corroborates the strong activations in the STG. A strong Group $\times$ Peristimulus Time effect was also observed, $F(6,78)=10.18, P<0.001$. The interaction was especially strong for all four time points from 9 to 18 seconds, $F(1,13)=12.30, P<0.001 ; F(1,13)=16.78, P<0.001 ; F(1,13)=$ $8.27, P<0.05 ; F(1,13)=19.34, P<0.001$. This suggests that, for this component, patients have a much higher peak than controls, suggesting hyperactivity in the networks involving the STG. The absence of a three-way interaction suggested that this hyperactivity for patients was independent of condition, and separate analyses for the generation and speech perception conditions confirmed significant Group $\times$ Peristimulus Time interactions for both, $F(1,13)=$ $4.18, P<0.001 ; F(1,13)=8.53, P<0.001$, respectively.

4. Discussion 
Dysfunction in the cognitive processes involved in the generation of private thoughts may contribute to the blurring of the ego boundaries in schizophrenia, either through reduced salience of top-down processes involved in generating thoughts, or rich bottom-up auditory qualities associated with generating thoughts. In the present study, the neural underpinnings of silent verbal thought generation and speech perception were investigated using fMRI. Functional connectivity analyses were performed using fMRI-CPCA. Six reliable functional networks were extracted, and related back to the experimental conditions by interpreting the associated predictor weights. As was expected from previous studies (Müller et al., 1997), two main types of networks were observed: one involved primarily in silent thought generation, which displayed strong dorsal anterior cingulate cortex (dACC) activations (Components 1 and 4), and another primarily involved in speech and voice perception, which displayed strong STG activations (Components 2, 5 and 6). One functional network was active in both conditions (Component 3, albeit with delayed peaks in the thought generation condition relative to speech perception), and correspondingly showed activations in both the dACC and STG. Group differences were observable on two functional networks: one reflecting hyperactivity in speech- and voice-selective cortical regions (e.g., bilateral STG) during both speech perception and silent verbal thought generation (Component 6), and another reflecting hyperactivity in a multiple demands (i.e., task-positive) network, which included Wernicke's area, during silent verbal thought generation (Component 1). Hyperactivation in functional networks involving speech- and voice-selective cortical regions during silent thought generation may contribute to the blurring of ego boundaries characteristic of schizophrenia.

All functional networks that showed greater activation during speech perception than during thought generation involved activation of the STG (i.e., Components 2, 5 and 6). However, only one (Component 6) differed between groups. Differences between patients and controls are likely to have led to Component 6 separating from Components 2 and 5 . 
Therefore, in order to understand how the results relate to schizophrenia, the spatial patterns of these three components, over and above their common STG activation, should be contrasted. Note that in addition to the STG activation, Component 2 visual cortex activation, and Component 5 involved the task-negative (default mode) network deactivations (Raichle et al., 2001; Fox et al., 2005). In contrast, Component 6 involved deactivations in regions normally associated with the task-positive or multiple demands network (Duncan and Owen, 2000; Fox et al., 2005), including subcortical structures, lateral prefrontal regions, and lateral superior parietal regions, although the dACC was notably absent. Recall that deactivation (negative loadings) refers to regions that decrease activity during task-on periods relative to task-off periods, and that activation (positive loadings) refers to regions that increase activity during task-on periods relative to task-off periods. Deactivation of task-positive regions is highly unusual for an attentionally demanding task, and for the other components (e.g., Component 3) we see activation in some of these regions in both conditions. This suggests hyper-intensity of a network involving activation of voice-selective cortical regions, and deactivation of regions normally involved in the allocation of attentional resources (but excluding the dACC), in both voice perception and silent thought generation in schizophrenia.

Deactivation of regions involved in allocation of attentional resources typically occurs only during task-off or self-reflective periods (Raichle et al., 2001; Fox et al., 2005). This may be particularly important for understanding inner/outer confusion, as hyper-intense cooperation between functional networks involved in self-reflection and those involved in perception of language suggests rich auditory qualities associated with self-reflective silent thoughts, contributing to inner/outer confusion. This suggests that a reduction in prefrontal cortex activation is indeed present for patients, a position shared with Frith's biological disconnection account (Frith, 1992, p. 84; McGuire et al., 1995; McGuire et al., 1996b); however, these results suggests that this reduction is attributable to more intense deactivation, 
as opposed to less intense activation, during silent thought. This set of results (1) is congruent with a number of existing accounts of inner-outer confusion in schizophrenia (Ford et al., 2007; Northoff and Qin, 2011), (2) can account for the observation of externalization errors (Seal et al., 2004; Ditman and Kuperberg, 2005; Woodward et al., 2006; Larøi and Woodward, 2007; Woodward et al., 2007) and reduced hits on self-generated items (reviewed by Waters et al., 2010) in source monitoring studies in schizophrenia (Woodward and Menon, in press), and (3) forms the basis of the "over-perceptualization" account of hallucinations (Allen et al., 2008).

Two components in this study were more active in the generation condition than the speech perception condition (Components 1 and 4), and involved the dACC. However, only one (Component 1) differed between the groups. Importantly, as mentioned above in reference to Component 6, differences between patients and controls likely caused Component 1 to emerge separately from Component 4, and it is important to compare the spatial patterns of these components beyond their common dACC activation. The most obvious difference is that Component 4 includes negative loadings corresponding to the tasknegative (i.e., default) network. This reciprocal pattern is commonly seen in attentionally demanding tasks (Fox et al., 2005; Metzak et al., 2011a; Metzak et al., 2011b), and since this did not differ between the groups, it will not be discussed further. Interestingly, Component 1 , which was hyperactive in patients, displayed activity in Wernicke's area whereas Component 4 did not. As with Component 6, this provides more evidence for hyperactivity of speech- and voice-selective cortical regions in schizophrenia.

One component, Component 3, did not differ between groups, and displayed a similar level of activity in both conditions. These were task-positive regions presumably involved in very general requirements for attentional resources that were equivalent in the two conditions, including primary sensory (visual and auditory) left prefrontal/dorsolateral prefrontal cortex 
(maintaining task instructions) and subcortical regions. The slight delay in the peak for the estimated BOLD signal associated with the thought generation condition relative to the speech perception condition is presumably due to generation processes taking slightly longer than perceptual processes to initiate.

One limitation of the current study is the small number of subjects. Although the standard errors in the plots of the estimated hemodynamic response shapes suggest that the functional networks were quite reliable, with a larger sample it would be possible to split the patients according to symptomatology, and to determine specific effects in hallucinating patients, or in those patients experiencing Schneiderian delusions involving alienation. Our hypothesis would be that the effects reported here would be increased with a pure sample of patients experiencing hallucinations or alienation symptoms. However, with the current dataset, such conclusions are not possible, and even the conclusion that these effects are present across the diagnostic category of schizophrenia must be considered preliminary due to the small sample size. A second limitation was that there is no direct method to check performance. However, given that group differences involved increased intensity for patients relative to controls, it seems unlikely that the results were influenced by patients being disengaged from the experimental tasks. A third limitation is that participants had familiarity (through training) with the words used for thought generation, but not for speech perception, which may have affected interpretation when the conditions were directly compared. (As mentioned above, rehearsing the speech perception material was not carried out as it would have increased the likelihood of self-generation of definitions during that condition.) Finally, unlike univariate methodologies, all significance testing for fMRI-CPCA take place at the level of standard repeated-measures ANOVAs, for which $P$ value corrections are not typically carried out. However, correction of the $P$ value for the repeated contrasts may be recommended, and it is worth noting that this set of results would remain unchanged if a more 
conservative $P$ value of $<0.01$ were used for the repeated contrasts instead of the typical $P<$ 0.05. It should also be noted that if a more conservative $P$ value of $<0.01$ were also used for the repeated measures ANOVAs, the Group effect on Component 1 would not survive.

\section{Conclusions}

In this study we provide preliminary evidence for hyperintensity of functional networks involving speech and voice-selective cortical regions (such as the STG and Wernicke's area) during silent thought generation in schizophrenia. This may contribute to the blurring of the ego boundaries and inner/outer confusions characteristic of this illness, but this must be confirmed in future work involving comparison of subsamples split on symptom profiles (Woodward and Menon, in press). 


\section{Acknowledgments}

LAR was supported by an Explora'Doc Grant from the Rhône-Alpes region. TSW was supported by a Scholar award from the Michael Smith Foundation for Health Research (MSFHR) and a New Investigator award from the Canadian Institutes of Health Research (CIHR). Operating costs were supported by a grant to TSW from the Mind Foundation of British Columbia. The data were submitted in partial fulfilment for the Ph.D. dissertation of LAR at the University of Grenoble, France. We sincerely thank Dr. Monica Baciu, Dr. Marc Sato, Dr. Simon McCarthy-Jones and Pr. Pascal Perrier for their helpful discussions, and John Paiement and Jennifer Riley with assistance in computer programming and manuscript preparation. 


\section{References}

Allen, P., Larøi, F., McGuire, P. K., Aleman, A., 2008. The hallucinating brain: A review of structural and functional neuroimaging studies of hallucinations. Neuroscience \& Biobehavioral Reviews 32, 175-191.

Annett, M. A., 1970. A classification of hand preference by association analysis. British Journal of Psychiatry 61, 303-321.

American Psychiatric Association, 2000. Diagnostic and statistical manual of mental disorders, Fourth edition, Text revision. Washington: American Psychiatric Association.

Belin, P., Zatorre, R. J., Lafaille, P., Ahad, P., Pike, B., 2000. Voice-selective areas in human auditory cortex. Nature 403, 309-312.

Binder, J. R., Frost, J. A., Hammeke, T. A., Bellgowan, P. S., Springer, J. A., Kaufman, J. N., Possing, E. T., 2000. Human temporal lobe activation by speech and nonspeech sounds. Cerebral Cortex 10, 512-528.

Buchsbaum, B. R., Olsen, R. K., Koch, P. F., Kohn, P., Kippenhan, J. S., Berman, K. F., 2005. Reading, hearing, and the planum temporale. Neuroimage 24, 444-454.

Cattell, R., 1966. The scree test for the number of factors. Multivariate Behavioural Research $1,245-276$.

Cattell, R., 1977. A comprehensive trial of the scree and KG criteria for determining the number of factors. Multivariate Behavioral Research 12, 289-325.

Coltheart, M., 1981. The MRC psycholinguistic database. Quarterly Journal of Experimental Psychology: Human Experimental Psychology 33A, 497-505.

Corporation, T. P., 2001. Manual for the Wechsler test of adult reading (WTAR). San Antonio, TX: Author. 
Curtis, V. A., Bullmore, E. T., Brammer, M. J., Wright, I. C., Williams, S. C., Morris, R. G., Sharma, T. S., Murray, R. M., McGuire, P. K., 1998. Attenuated frontal activation during a verbal fluency task in patients with schizophrenia. American Journal of Psychiatry 155, 1056-1063.

Ditman, T., Kuperberg, G. R., 2005. A source-monitoring account of auditory verbal hallucinations in patients with schizophrenia. Harvard Review of Psychiatry 13, 280299.

Duncan, J., Owen, A. M., 2000. Common regions of the human frontal lobe recruited by diverse cognitive demands. Trends in Neurosciences 23, 475-483.

Ford, J. M., Roach, B. J., Faustman, W. O., Mathalon, D. H., 2007. Synch before you speak: Auditory hallucinations in schizophrenia. American Journal of Psychiatry 164, 458466.

Fox, M. D., Snyder, A. Z., Vincent, J. L., Corbetta, M., Van Essen, D. C., Raichle, M. E., 2005. The human brain is intrinsically organized into dynamic, anticorrelated functional networks. Proceedings of the National Academy of Sciences USA 102, 9673-9678.

Friedman, L., Kenny, J. T., Wise, A. L., Wu, D., Stuve, T. A., Miller, D. A., Jesberger, J. A., Lewin, J. S., 1998. Brain activation during silent word generation evaluated with functional MRI. Brain and Language 64, 231-256.

Frith, C. D., 1992. The cognitive neuropsychology of schizophrenia. Hillsdale, NJ: Lawrence Erlbaum.

Frith, C. D., Friston, K. J., Herold, S., Silbersweig, P., Fletcher, C., Cahill, R. J., Dolan, R., Frackowiak, S. J., Liddle, P. F., 1995. Regional brain activity in chronic schizophrenic patients during the performance of a verbal fluency task. British Journal of Psychiatry 167, 343-349. 
Frith, C. D., Friston, K. J., Liddle, P. F., Frackowiak, R. S., 1991. A PET study of word finding. Neuropsychologia 29, 1137-1148.

Hickok, G., Poeppel, D., 2007. The cortical organization of speech processing. Nature Reviews. Neuroscience 8, 393-402.

Kaufman, A. S., Kaufman, N. L., 1997. Kaufman brief intelligence test second edition (kbit 2) manual. Minneapolis, MN: NCS Pearson, Inc.

Larøi, F., Woodward, T. S., 2007. Hallucinations from a cognitive perspective. Harvard Review of Psychiatry 15, 109-117.

Liddle, P. F., Ngan, E. T. N., Duffield, G., Kho, K., Warren, A. J., 2002. The signs and symptoms of psychotic illness: A rating scale. British Journal of Psychiatry 180, $45-$ 50.

McGuire, P. K., Silbersweig, D. A., Murray, R. M., David, A. S., Frackowiak, R. S., Frith, C. D., 1996a. Functional anatomy of inner speech and auditory verbal imagery. Psychological Medicine 26, 29-38.

McGuire, P. K., Silbersweig, D. A., Wright, I., Murray, R. M., David, A. S., Frackiowack, R. S. J., Frith, C. D., 1995. Abnormal monitoring of inner speech: A physiological basis for auditory hallucinations. The Lancet 346, 596-600.

McGuire, P. K., Silbersweig, D. A., Wright, I., Murray, R. M., Frackowiak, R. S., Frith, C. D., 1996b. The neural correlates of inner speech and auditory verbal imagery in schizophrenia: Relationship to auditory verbal hallucinations. British Journal of Psychiatry 169, 148-159.

Metzak, P. D., Feredoes, E., Takane, Y., Wang, L., Weinstein, S., Cairo, T., Ngan, E. T. C., Woodward, T. S., 2011a. Constrained principal component analysis reveals functionally connected load-dependent networks involved in multiple stages of working memory. Human Brain Mapping 32, 856-871. 
Metzak, P. D., Riley, J., Wang, L., Whitman, J. C., Ngan, E. T. C., Woodward, T. S., 2011 b. Decreased efficiency of task-positive and task-negative networks during working memory in schizophrenia. Schizophrenia Bulletin, 10.1093/schbul/sbq1154.

Müller, R. A., Rothermel, R. D., Behen, M. E., Muzik, O., Mangner, T. J., Chugani, H. T., 1997. Receptive and expressive language activations for sentences: A PET study. Neuroreport 8, 3767-3770.

Northoff, G., Qin, P., 2011. How can the brain's resting state activity generate hallucinations? A 'resting state hypothesis' of auditory verbal hallucinations. Schizophrenia Research $127,202-214$.

Raichle, M. E., MacLeod, A. M., Snyder, A. Z., Powers, W. J., Gusnard, D. A., Shulman, G. L., 2001. A default mode of brain function. Proc Natl Acad Sci U S A 98, 676-682.

Seal, M. L., Aleman, A., McGuire, P. K., 2004. Compelling imagery, unanticipated speech and deceptive memory: Neurocognitive models of auditory verbal hallucinations in schizophrenia. Cognitive Neuropsychiatry 9, 43-72.

Serences, J. T., 2004. A comparison of methods for characterizing the event-related bold timeseries in rapid fMRI. Neuroimage 21, 1690-1700.

Sheehan, D. V., Lecrubier, Y., Sheehan, K. H., Amorim, P., Janavs, J., Weiller, E., Hergueta, T., Baker, R., Dunbar, G. C., 1998. The mini-international neuropsychiatric interview (m.I.N.I.): The development and validation of a structured diagnostic psychiatric interview for DSM-IV and ICD-10. Journal of Clinical Psychiatry 59 (Suppl. 20), 2233.

Takane, Y., Hunter, M. A., 2001. Constrained principal component analysis: A comprehensive theory. Applicable Algebra in Engineering, Communication and Computing 12, 391-419. 
Takane, Y., Shibayama, T., 1991. Principal component analysis with external information on both subjects and variables. Psychometrika 56, 97-120.

Waters, F., Woodward, T. S., Allen, P., Aleman, A., Sommer, I., 2010. Self-recognition deficits in schizophrenia patients with auditory hallucinations: A meta-analysis of the literature. Schizophrenia Bulletin DOI:10.1093/schbul/sbq144.

Woodward, T. S., Menon, M., in press. Misattribution models (ii): Source monitoring in hallucinating schizophrenia subjects. In R. Jardri, D. Pins, A. Cachia \& P. Thomas (Eds.), The neuroscience of hallucinations: Springer.

Woodward, T. S., Menon, M., Hu, X., Keefe, R. S. E., 2006. Optimization of a multinomial model for investigations of hallucinations and delusions with source monitoring. Schizophrenia Research 85, 106-112.

Woodward, T. S., Menon, M., Whitman, J. C., 2007. Source monitoring biases associated with hallucinations in schizophrenia. Cognitive Neuropsychiatry 12, 477-494.

Zatorre, R. J., Evans, A. C., Meyer, E., Gjedde, A., 1992. Lateralization of phonetic and pitch discrimination in speech processing. Science 256, 846-849. 
Figure Captions

Figure 1. Timeline of the experimental procedure. Two functional scan runs of 9 minutes and 25 seconds each were completed, and stimuli in each condition (silent verbal thought generation and speech perception) were grouped in blocks consisting of fifteen trials in each run. In the silent verbal thought generation condition, participants were required to mentally generate a simple definition of a word, and in the speech perception condition, participants listened to a simple definition. The first screen in each block displayed the instructions for that experimental condition, which remained on the screen for 15 seconds. The first trial of the specified condition was subsequently displayed for two seconds, and was followed by a blank screen (three seconds) and then by the ISI screen prior to the onset of the second trial in that condition. The distribution of the duration of the ISI was exponential, optimizing the deconvolution of the BOLD signal (Serences, 2004) (mean=4.46s; range=2s, 4, 6, 8, 16, 20s). Although the stimuli were presented in blocks, the analyses were carried out using an eventrelated model in order to estimate trial-dependent HDR shapes.

Figure 2. Images, plots of predictor weights, and plots of BOLD signal for Components 1-6. The dominant $5 \%$ of component loadings are displayed, with positive component loadings displayed in red and negative component loadings in blue. The mean FIR-based predictor weights are plotted as a function of peristimulus time and condition for the control group and the schizophrenia group (error bars are standard errors). The predictor weights at the first point of peristimulus time are adjusted to zero and all other values scaled accordingly for each subject. 


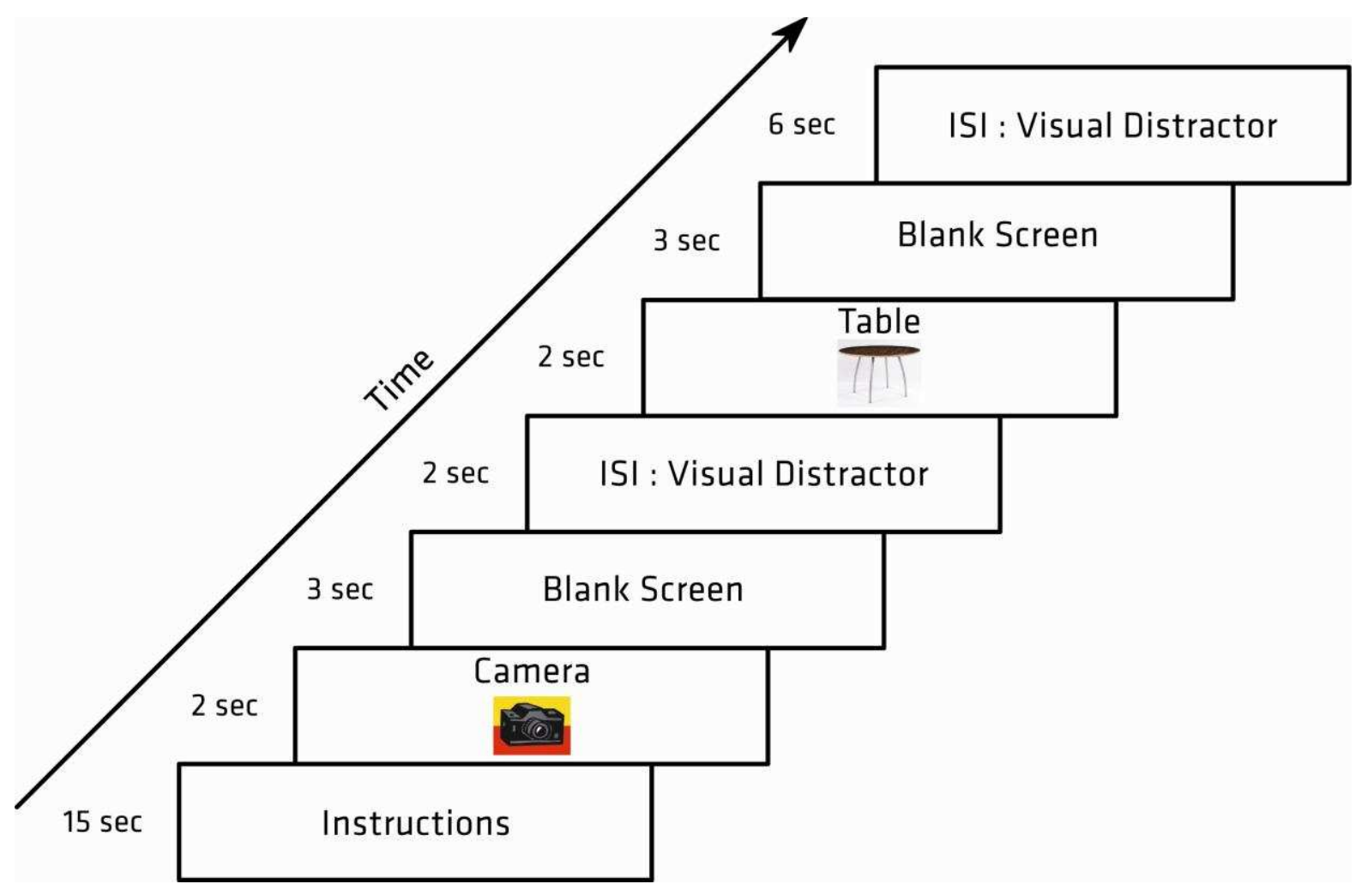



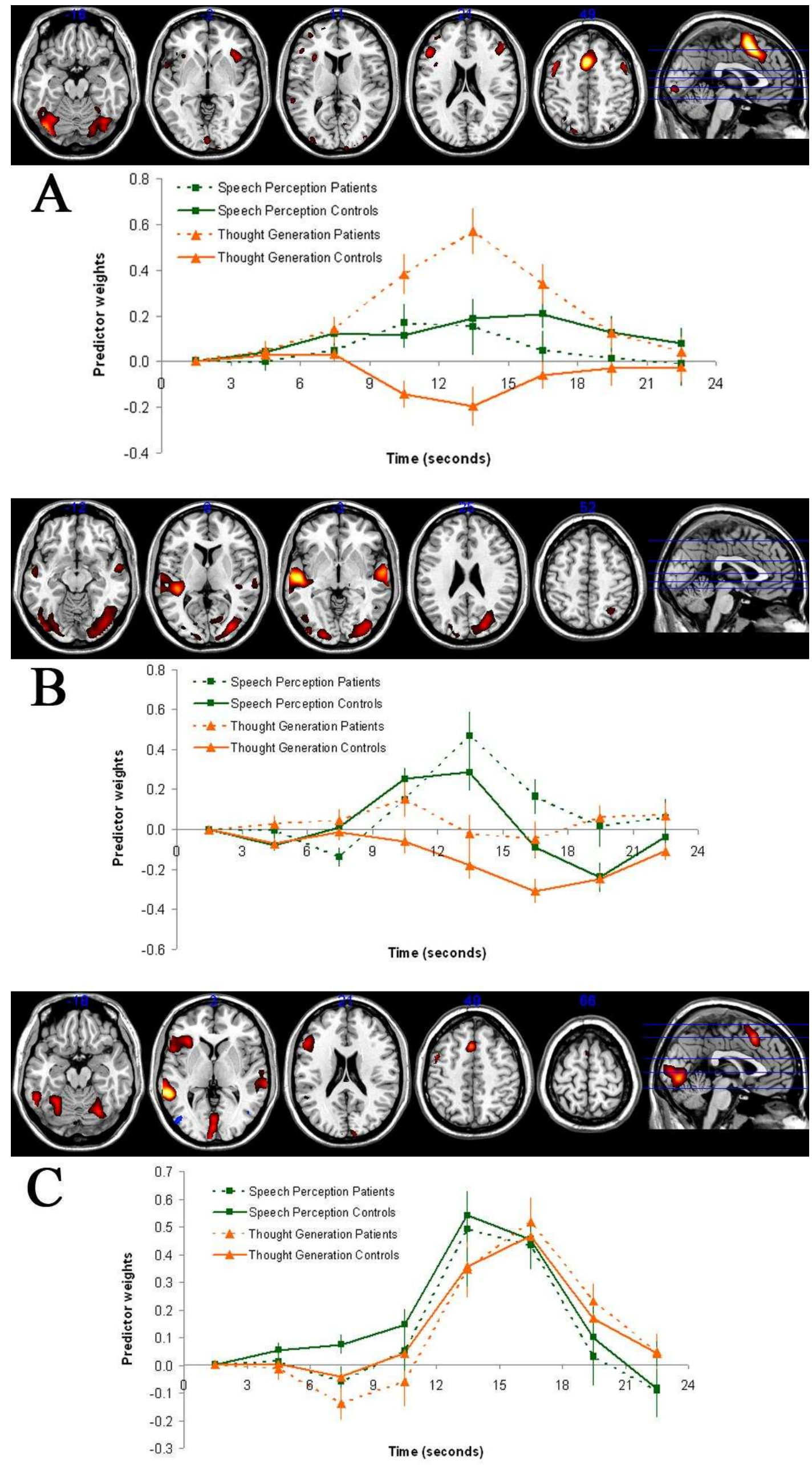

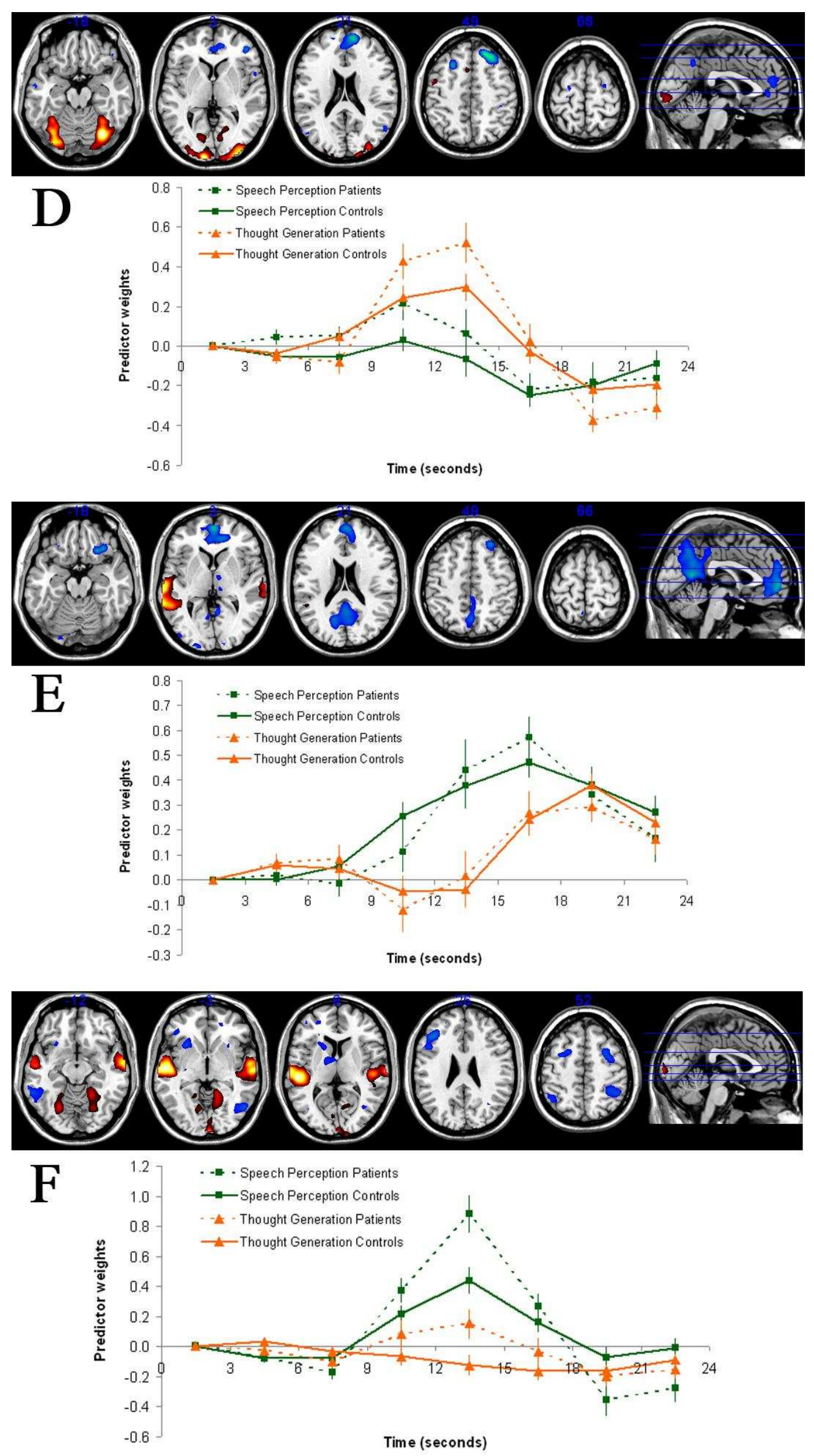